\title{
Synthesis and biological activity of myricetin derivatives containing 1,3,4-thiadiazole scaffold
}

\author{
Xinmin Zhong ${ }^{1 \dagger}$, Xiaobin Wang ${ }^{1,2+}$, Lijuan Chen ${ }^{1}$, Xianghui Ruan ${ }^{1}$, Qin $\mathrm{Li}^{1}$, Juping Zhang ${ }^{1}$, Zhuo Chen ${ }^{1}$ \\ and Wei Xue ${ }^{1 *}$
}

\begin{abstract}
Background: Myricetin and 1,3,4-thiadiazole derivatives were reported to exhibit favorable antiviral and antibacterial activities. Aiming to discover novel myricetin analogues with potent activities, a series of novel myricetin derivatives containing 1,3,4-thiadiazole moiety were synthesized, and their antibacterial and antiviral activities were evaluated.

Result: Bioassay results indicated that some target compounds exhibited potential antibacterial and antiviral activities. Among them, compounds $\mathbf{2}, \mathbf{3 a}, \mathbf{3 b}, \mathbf{3} \mathbf{d}, \mathbf{3 f}, \mathbf{3 i}, \mathbf{3 m}$ and $\mathbf{3 p}$ exhibited excellent antibacterial activities against Xanthomonas oryzae pv. Oryzae (Xoo), with $\mathrm{EC}_{50}$ values of $42.7,38.6,20.8,12.9,22.7,27.3,18.3$ and $29.4 \mu \mathrm{g} / \mathrm{mL}$, respectively, which were better than that of thiadiazole-copper $(94.9 \mu \mathrm{g} / \mathrm{mL})$. Compounds $\mathbf{3 b}, \mathbf{3 d}, \mathbf{3 e}, \mathbf{3 f}, \mathbf{3 i}$ and $\mathbf{3 0}$ showed good antibacterial activities against Ralstonia solanacearum (Rs), with $\mathrm{EC}_{50}$ values of $37.9,72.6,43.6,59.6,60.6$ and $39.6 \mu \mathrm{g} / \mathrm{mL}$, respectively, which were superior to that of thiadiazole-copper $(131.7 \mu \mathrm{g} / \mathrm{mL})$. In addition, compounds 3d, 3f, 3i and $\mathbf{3 m}$ showed better curative activities against tobacco mosaic virus (TMV), with $\mathrm{EC}_{50}$ values of 152.8, 99.7, 127.1 , and $167.3 \mu \mathrm{g} / \mathrm{mL}$, respectively, which were better than that of ningnanmycin $(211.1 \mu \mathrm{g} / \mathrm{mL})$.

Conclusions: A series of myricetin derivatives containing 1,3,4-thiadiazole scaffold were synthesized, and their antibacterial activities against $X_{O O}$ and $R s$ and their antiviral activity against TMV were evaluated. Bioassays indicated that some target compounds exhibited potential antibacterial and antiviral activities. These results indicated this kind of myricetin analogues could be further studied as potential alternative templates in the search for novel antibacterial and antiviral agents.
\end{abstract}

Keywords: Myricetin, 1,3,4-thiadiazole, Antibacterial activity, Antiviral activity

\section{Background}

The rational use of agrochemicals plays a pivotal role in agricultural production by effectively controlling plant diseases $[1,2]$. Unfortunately, the application of traditional pesticides is greatly limited due to their negative impacts on the environment and the rapid emergence of resistance $[2,3]$. Therefore, searching for high-efficiency

\footnotetext{
*Correspondence: wxue@gzu.edu.cn

${ }^{\dagger}$ Xinmin Zhong and Xiaobin Wang contributed equally to this work

1 State Key Laboratory Breeding Base of Green Pesticide and Agricultural Bioengineering, Key Laboratory of Green Pesticide and Agricultural Bioengineering, Ministry of Education, Guizhou University, Guiyang 550025, China

Full list of author information is available at the end of the article
}

and environmentally friendly agrochemicals remains an arduous challenge in pesticide chemistry $[1,4]$. In this process, natural products and their derivatives with new modes of action have been developed as pesticides that are safe to the environment $[5,6]$.

As one of important natural products in medicinal chemistry, myricetin was reported to exhibit extensive bioactivities including antibacterial [7], antiviral [8], anticancer [9], anti-inflammatory [10], antioxidant [11], and hypoglycemic activities [12]. Our previous study extracted a mixture containing myricetin from the bark of Toona sinensis and found it to exhibit moderate antiviral activity against tobacco mosaic virus (TMV) [13]. Using natural myricetin as the lead molecule, some 
myricetin derivatives bearing Schiff-base moiety, which displayed good inhibitory activity against telomerase and excellent anticancer activity against human breast cancer cells MDA-MB-231, were synthesized by Xue et al. [14]. Furthermore, the acceptable antibacterial activities against Xanthomonas oryzae pv. oryzae (Xoo) and Ralstonia solanacearum (Rs) of myricetin derivatives containing acidamide moiety were also recently reported by us [15]. Obviously, myricetin derivatives as possible active ingredients play a key role in the searching for novel agrochemicals and pharmaceuticals (Fig. 1).

1,3,4-Thiadiazoles, which represent important nitrogenous heterocycles in medicinal chemistry, have attracted much attentions because of their various pharmacological activities, including antibacterial [16], antifungal [17], antiviral [18], anticonvulsant [19], anxiolytic [20], antinociceptive [21] and anticancer [22] activities. Among the above biological activities, acceptable antibacterial and antiviral activities displayed by 1,3,4-thiadiazoles have been reported well by chemists in recent years. For example, Li et al. [23] found that some 1,3,4-thiadiazole sulfone derivatives exhibited satisfactory antibacterial activities against rice bacterial leaf blight and leaf streak. Recently, we also found some 1,3,4-thiadiazole derivatives bearing 1,4-pentadiene-3-one moiety to exhibit remarkable antiviral activities against plant viruses [24].

Considering these above results, we speculated that introducing 1,3,4-thiadiazole fragment into myricetin might generate novel lead compounds with greater biological activities. Thus, a series of myricetin derivatives containing 1,3,4-thiadiazole scaffold were synthesized (Scheme 1), and their antibacterial activities against Xoo and $R s$ and their antiviral activity against TMV were evaluated.

\section{Results and discussion Chemistry}

A series of myricetin derivatives containing thiadiazole moiety were successfully prepared in two steps in our current work. All of the target compounds $\mathbf{2}, \mathbf{3 a}-\mathbf{3 q}$ were characterized by infrared spectrum (IR), nuclear magnetic resonance (NMR) spectroscopy, and high resolution mass spectrum (HRMS) analysis. The IR spectral data of compounds $\mathbf{2}, \mathbf{3 a}-\mathbf{3 q}$ showed characteristic frequencies at 1723-1709 $\mathrm{cm}^{-1}$ and $1640-1621 \mathrm{~cm}^{-1}$, which are assigned to the characteristic vibrations of $\mathrm{C}=\mathrm{O}$ and $\mathrm{C}=\mathrm{N}-$, respectively. In the ${ }^{1} \mathrm{H}$ NMR spectra, the characteristic $-\mathrm{CH}_{2}-$ groups between myricetin scaffold and 1,3,4-thiadiazole heterocycle was observed
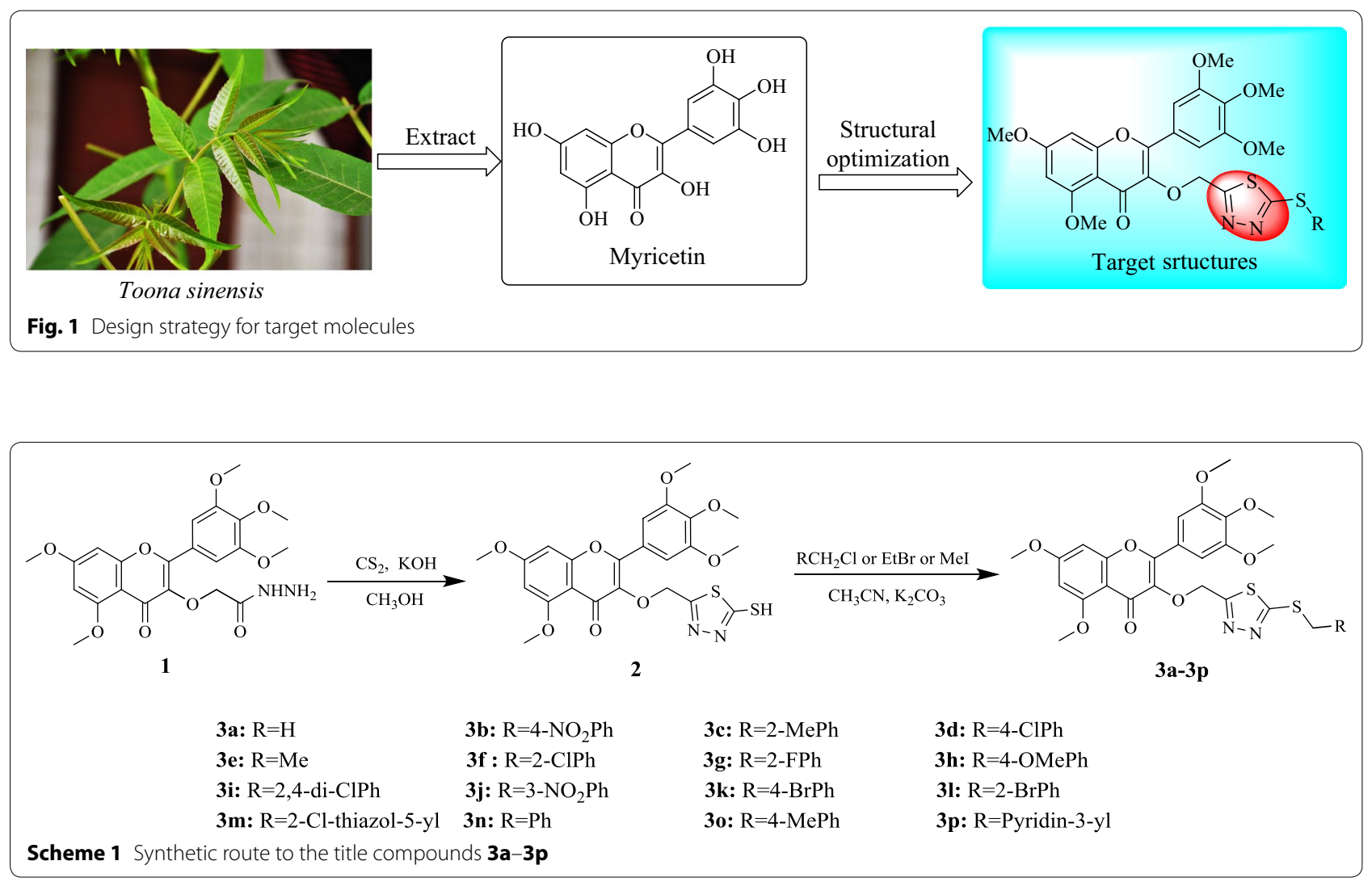
as a signal at approximately $5.27-5.21 \mathrm{ppm}$. The chemical shifts at 165.59-161.63 and 161.70-154.04 ppm in the ${ }^{13} \mathrm{C}$ NMR spectra confirmed the existence of $\mathrm{C}=\mathrm{O}$ and $\mathrm{C}=\mathrm{N}$-groups, respectively.

\section{Antibacterial activity screening of the title compounds against Xac and $R s$ in vitro}

Using Ralstonia solanacearum (strain MR111, Guizhou University, China) and Xanthomonas oryzae pv. oryzae (strain PXO99A, Nanjing Agricultural University, China) as the tested bacterial strains, the antibacterial activities of title compounds have been evaluated by the turbidimeter test $[1,3,4,6]$, and the commercial agent thiadiazole-copper was tested as the control. Some compounds with good antibacterial activity against $X o o$ and $R s$ were tested at five double-declining concentrations (100, 50, $25,12.5$ and $6.25 \mu \mathrm{g} / \mathrm{mL}$ ) to obtain the corresponding $\mathrm{EC}_{50}$ values.

The title compounds $(\mathbf{2}, \mathbf{3 a}-\mathbf{3 q})$ were evaluated for antibacterial activities against Xoo and Rs in vitro. Results in Table 1 indicated that most synthesized compounds exhibited appreciable antibacterial activities against Xoo and $R s$. For example, compounds $\mathbf{2}$, 3a, 3b, 3d, 3f, 3i, 3m and 3p showed excellent antibacterial activities against $X o o$ at $100 \mu \mathrm{g} / \mathrm{mL}$, with inhibition rates of 84.5, 84.9, 99.6, $87.3,77.5,84.5,99.3$ and $84.3 \%$, respectively, which were better than that of thiadiazole-copper (52.3\%). The inhibition rates of compounds $\mathbf{2}, \mathbf{3 a}, \mathbf{3} \mathbf{b}, \mathbf{3 d}, \mathbf{3 \mathbf { f }}, \mathbf{3 \mathbf { i }}, \mathbf{3} \mathbf{m}$ and 3 p against $X o o$ at $50 \mu \mathrm{g} / \mathrm{mL}$ were 54.6, 60.1, 65.2, 90.7, 82.6, 68.2, 80.8 and $71.2 \%$, respectively, which were better than that of thiadiazole-copper (28.7\%). Additionally, compounds 3b, 3d, 3e, 3f, 3i and 3o demonstrated good antibacterial activities against $R s$ at $100 \mu \mathrm{g} / \mathrm{mL}$, with inhibition rates of 81.4, 64.3, 75.7, 69.3, 64.3 and $65.4 \%$, respectively, which were superior to that of thiadiazolecopper (46.7\%). Compounds 3b, 3d, 3e, 3f, 3i and $3 \mathbf{s o}$ showed good antibacterial activities against $R s$ at $50 \mu \mathrm{g} /$ $\mathrm{mL}(60.2,30.4,65.5,40.5,52.2$ and $52.1 \%$, respectively), which were better than thiadiazole-copper (32.2\%).

To further understand antibacterial activity of synthesized compounds, the $\mathrm{EC}_{50}$ values of some target compounds, which exhibited better antibacterial activities against Xoo and Rs than thiadiazole-copper, were calculated and summarized in Table 2. Notably, compounds 2, 3a, 3b, 3d, 3f, 3i, 3m and 3p exhibited excellent antibacterial activities against Xoo, with $\mathrm{EC}_{50}$ values of 42.7, $38.6,20.8,12.9,22.7,27.3,18.3$ and $29.4 \mu \mathrm{g} / \mathrm{mL}$, respectively, which were better than that of thiadiazole-copper $(94.9 \mu \mathrm{g} / \mathrm{mL})$. Meanwhile, compounds 3b, 3d, 3e, 3f, 3i and 3o showed remarkable antibacterial activities against $R s$, with $\mathrm{EC}_{50}$ values of 37.9, 72.6, 43.6, 59.6, 60.6 and

Table 1 Inhibition effect of the compounds 4, 5a-5q against Xoo and $R s$

\begin{tabular}{|c|c|c|c|c|c|}
\hline \multirow[t]{2}{*}{ Compd. } & \multirow[t]{2}{*}{$\mathbf{R}$} & \multicolumn{2}{|l|}{ Xoo } & \multicolumn{2}{|l|}{ Rs } \\
\hline & & $100 \mu \mathrm{g} / \mathrm{mL}$ & $50 \mu \mathrm{g} / \mathrm{mL}$ & $100 \mu \mathrm{g} / \mathrm{mL}$ & $50 \mu \mathrm{g} / \mathrm{mL}$ \\
\hline 2 & - & $84.5 \pm 3.9$ & $54.6 \pm 8.5$ & 46.5. \pm 9.7 & $28.1 \pm 7.8$ \\
\hline $3 a$ & $\mathrm{H}$ & $84.9 \pm 5.8$ & $60.1 \pm 2.5$ & $36.0 \pm 2.6$ & $32.4 \pm 6.1$ \\
\hline $\mathbf{3 b}$ & $4-\mathrm{NO}_{2} \mathrm{Ph}$ & $81.4 \pm 4.6$ & $65.2 \pm 9.0$ & $81.5 \pm 6.7$ & $60.2 \pm 6.9$ \\
\hline $3 c$ & 2-MePh & $47.2 \pm 1.5$ & $25.9 \pm 3.7$ & $49.3 \pm 6.7$ & $30.3 \pm 3.8$ \\
\hline $3 d$ & $4-\mathrm{ClPh}$ & $99.6 \pm 0.1$ & $90.7 \pm 4.0$ & $64.3 \pm 8.8$ & $30.4 \pm 4.1$ \\
\hline $3 e$ & Me & $58.2 \pm 5.1$ & $27.4 \pm 5.4$ & $75.7 \pm 8.1$ & $65.5 \pm 9.9$ \\
\hline $3 f$ & $2-\mathrm{ClPh}$ & $87.3 \pm 2.5$ & $82.6 \pm 2.6$ & $69.3 \pm 0.8$ & $46.5 \pm 9.1$ \\
\hline $3 \mathbf{g}$ & $2-\mathrm{FPh}$ & $79.7 \pm 3.6$ & $21.0 \pm 4.9$ & $45.2 \pm 5.9$ & $38.3 \pm 2.4$ \\
\hline 3h & 4-OMePh & $37.3 \pm 6.2$ & $15.5 \pm 8.9$ & $28.1 \pm 7.6$ & $27.1 \pm 6.0$ \\
\hline $3 \mathbf{i}$ & 2,4-di-CIPh & $77.5 \pm 1.4$ & $68.2 \pm 5.4$ & $64.3 \pm 6.1$ & $52.1 \pm 2.8$ \\
\hline $\mathbf{3 j}$ & $3-\mathrm{NO}_{2} \mathrm{Ph}$ & $30.0 \pm 1.2$ & $79.8 \pm 9.7$ & $45.2 \pm 8.3$ & $31.1 \pm 4.3$ \\
\hline $3 \mathbf{k}$ & 4-BrPh & $47.3 \pm 4.7$ & $23.3 \pm 7.5$ & $26.4 \pm 2.6$ & $10.7 \pm 1.6$ \\
\hline 31 & $2-\mathrm{BrPh}$ & $50.7 \pm 1.9$ & $31.6 \pm 4.5$ & $24.0 \pm 4.7$ & $16.2 \pm 0.7$ \\
\hline $3 m$ & 2-Cl-thiazol-5-yl & $99.4 \pm 3.9$ & $80.8 \pm 3.7$ & $26.3 \pm 3.2$ & $25.0 \pm 6.6$ \\
\hline $3 n$ & $\mathrm{Ph}$ & $38.3 \pm 4.5$ & $17.7 \pm 0.1$ & $45.3 \pm 5.6$ & $44.7 \pm 5.1$ \\
\hline 30 & 4-MePh & $52.6 \pm 3.3$ & $37.6 \pm 5.5$ & $65.4 \pm 1.7$ & $52.1 \pm 5.7$ \\
\hline $3 p$ & Pyridin-3-yl & $84.3 \pm 3.8$ & $71.2 \pm 5.3$ & $38.0 \pm 6.2$ & $12.8 \pm 6.0$ \\
\hline Myricetin $^{a}$ & - & $40.1 \pm 8.3$ & $21.0 \pm 5.6$ & $28.6 \pm 2.2$ & $17.5 \pm 3.3$ \\
\hline Thiadiazole-copper & - & $52.4 \pm 2.0$ & $28.7 \pm 4.1$ & $46.7 \pm 2.0$ & $32.2 \pm 2.1$ \\
\hline
\end{tabular}

Average of three replicates

a Thiadiazole-copper and myricetin were used for comparison of antibacterial activity 
Table $2 \mathrm{EC}_{50}$ values of target compounds against $X o 0$ and $R s$

\begin{tabular}{|c|c|c|c|c|c|c|}
\hline \multirow[t]{2}{*}{ Compd. } & \multicolumn{3}{|l|}{ Xoo } & \multicolumn{3}{|l|}{ Rs } \\
\hline & Regression equation & $\mathbf{r}$ & $E C_{50}(\mu \mathrm{g} / \mathrm{mL})$ & Regression equation & $r$ & $E C_{50}(\mu \mathrm{g} / \mathrm{mL})$ \\
\hline 2 & $y=2.513 x+0.902$ & 0.99 & $42.7 \pm 2.6$ & / & / & / \\
\hline $3 a$ & $y=2.885 x+0.454$ & 0.99 & $38.6 \pm 1.4$ & / & / & / \\
\hline $3 \mathbf{b}$ & $y=1.199 x+3.420$ & 0.99 & $20.8 \pm 3.6$ & $y=2.685 x+0.762$ & 0.99 & $37.9 \pm 1.0$ \\
\hline 3d & $y=2.328 x+2.418$ & 0.97 & $12.9 \pm 5.8$ & $y=2.770 x-0.154$ & 0.99 & $72.6 \pm 1.6$ \\
\hline $3 e$ & / & / & / & $y=2.485 x+0.925$ & 0.98 & $43.6 \pm 3.8$ \\
\hline $3 f$ & $y=1.982 x+2.314$ & 0.98 & $22.7 \pm 3.6$ & $y=3.004 x-0.332$ & 0.99 & $59.6 \pm 2.0$ \\
\hline $3 \mathbf{i}$ & $y=1.401 x+2.989$ & 0.99 & $27.3 \pm 1.8$ & $y=2.365 x+0.786$ & 0.99 & $60.6 \pm 2.1$ \\
\hline $3 m$ & $y=2.723 x+1.565$ & 0.98 & $18.3 \pm 3.6$ & / & / & / \\
\hline $3 p$ & $y=2.058 x+1.979$ & 0.99 & $29.4 \pm 1.0$ & / & / & / \\
\hline 30 & / & / & / & $y=1.017 x+3.375$ & 0.96 & $39.6 \pm 5.3$ \\
\hline Thiadiazole-copper ${ }^{\mathrm{a}}$ & $y=1.999 x+1.047$ & 0.99 & $94.9 \pm 2.2$ & $y=0.930 x+3.028$ & 0.98 & $131.7 \pm 2.9$ \\
\hline
\end{tabular}

Average of three replicates

a The commercial agricultural antibacterial agent thiadiazole-copper was used for comparison of antibacterial activity

$39.6 \mu \mathrm{g} / \mathrm{mL}$, respectively, which were superior to that of thiadiazole-copper $(131.7 \mu \mathrm{g} / \mathrm{mL})$.

The inhibitory rates in Tables 1 and 2 indicated that most synthesized compounds bearing the same substituted fragment were found to exhibit better antibacterial activity against Xoo than $R s$. For example, the $\mathrm{EC}_{50}$ values of title compounds $\mathbf{3 b}, \mathbf{3 d}, \mathbf{3} \mathbf{f}$ and $3 \mathbf{i}$ against $X o o$ were respectively $20.8,12.9,22.7$ and $27.3 \mu \mathrm{g} / \mathrm{mL}$, which were better than that against Rs $(37.9,72.6,59.6$ and 60.6 $\mu \mathrm{g} / \mathrm{mL}$, respectively). The antibacterial results in Tables 1 and 2 also indicated that the different groups on $\mathrm{R}$ had significant effects on the antibacterial activity of the target compounds. Obviously, the presence of heterocycles can effectively enhance the antibacterial activity against Xoo. As examples of this phenomenon, the compounds $3 \mathbf{m}$ and $3 \mathbf{p}$, which contain respectively 2 -Cl-thiazol5 -yl and pyridin-3-yl groups, exhibited fine antibacterial activities against $X o o$ at $50 \mu \mathrm{g} / \mathrm{mL}$, with the inhibition rates of 80.8 and $71.2 \%$, respectively, which were superior to that of thiadiazole-copper (28.7\%). Meanwhile, when $\mathrm{R}$ was substituted with $4-\mathrm{NO}_{2} \mathrm{Ph}, 4-\mathrm{ClPh}, 2-\mathrm{ClPh}$ and 2,4-di-ClPh groups, the corresponding compounds 3b, 3d, 3f and 3i exhibit remarkable antibacterial activities against $\mathrm{Xoo}$, with the $\mathrm{EC}_{50}$ values of $20.8,12.9,22.7$ and $27.3 \mu \mathrm{g} / \mathrm{mL}$, respectively, which were better than that of thiadiazole-copper $(94.9 \mu \mathrm{g} / \mathrm{mL})$.

\section{Antiviral activity screening of the title compounds against TMV in vivo}

Using growing $N$. tobacum $L$. leaves at the same age as the test subjects, the curative and protective activities against TMV were evaluated based on the half-leaf blight spot method [25-27], and the commercial agent ningnanmycin was tested as the control under the same conditions. The antiviral activity against TMV in vivo at $500 \mu \mathrm{g} / \mathrm{mL}$ was listed in Tables 3 and 4 . The preliminary bioassays results indicated that the inhibitory rates of title compounds against TMV at $500 \mu \mathrm{g} / \mathrm{mL}$ ranged from 18.2 to $68.4 \%$ in terms of their curative activity, and ranged from 21.5 to $60.8 \%$ in terms of their protective activity. Among them, the inhibitory rates of compounds 3d, 3f, 3i and $\mathbf{3 m}$ in curative activity were 59.8, 68.4, 66.8 and $57.1 \%$, respectively, which were better than that of ningnanmycin (51.8\%). Moreover, compounds $3 \mathbf{c}, 3 \mathbf{i}$ and 3m were found to exhibit significant protective activities (58.4, 60.8 and 56.7\%, respectively), which were similar to ningnanmycin (58.3\%).

To further understand antiviral activity of synthesized compounds, the $\mathrm{EC}_{50}$ values of $\mathbf{3 d}, \mathbf{3 f}, \mathbf{3 i}$ and $\mathbf{3 m}$ were calculated and summarized in Table 4. Notably, the $\mathrm{EC}_{50}$ values of $\mathbf{3 d}$, 3f, $\mathbf{3 i}$ and $\mathbf{3 m}$ were respectively $152.8,99.7$, 127.1 and $167.3 \mu \mathrm{g} / \mathrm{mL}$, which were better than that of ningnanmycin $(211.1 \mu \mathrm{g} / \mathrm{mL})$.

The antiviral results in Tables 3 and 4 indicated that most of synthesized compounds bearing the same substituted fragment exhibited better protective activity than curative activity against TMV. Meanwhile, Results in Tables 3 and 4 also indicated that the different groups on $\mathrm{R}$ had significant effects on the anti-TMV activity of the target compounds. Obviously, the presence of benzyl chloride groups can effectively enhance the curative activity of title compounds against TMV. For example, compounds 3d, 3f, $\mathbf{3 i}$ and $\mathbf{3 m}$, which contain respectively 2-ClPh, 4-ClPh, 2,4-di-ClPh and 2-Cl-thiazol-5-yl groups, exhibited excellent curative activities against TMV, with the $\mathrm{EC}_{50}$ values of $152.8,99.7,127.1$ and $167.3 \mu \mathrm{g} / \mathrm{mL}$, respectively, which were better than that of ningnanmy$\operatorname{cin}(211.1 \mu \mathrm{g} / \mathrm{mL})$. Furthermore, when the $\mathrm{R}$ was $2-\mathrm{MePh}$, 
Table 3 Antiviral activities of the title compounds against TMV in vivo at $500 \mu \mathrm{g} / \mathrm{mL}$

\begin{tabular}{|c|c|c|c|c|c|}
\hline Compd. & Curative activity (\%) & Protection activity (\%) & Compd. & Curative activity (\%) & Protection activity (\%) \\
\hline 2 & $18.2 \pm 7.3$ & $21.5 \pm 9.1$ & $\mathbf{3 j}$ & $28.7 \pm 3.8$ & $39.4 \pm 3.1$ \\
\hline $3 a$ & $46.7 \pm 5.2$ & $50.3 \pm 9.3$ & $3 \mathbf{k}$ & $28.0 \pm 8.6$ & $33.0 \pm 7.5$ \\
\hline $3 \mathbf{b}$ & $53.8 \pm 9.0$ & $54.1 \pm 9.4$ & 31 & $33.9 \pm 9.4$ & $34.2 \pm 5.4$ \\
\hline $3 c$ & $37.0 \pm 9.1$ & $58.4 \pm 1.0$ & $3 m$ & $57.1 \pm 9.6$ & $56.7 \pm 8.2$ \\
\hline 3d & $59.8 \pm 9.2$ & $54.3 \pm 9.0$ & $3 n$ & $48.4 \pm 5.9$ & $42.1 \pm 7.1$ \\
\hline $3 e$ & $28.7 \pm 8.3$ & $35.4 \pm 5.1$ & 30 & $50.8 \pm 3.6$ & $47.3 \pm 2.9$ \\
\hline $3 f$ & $68.4 \pm 7.4$ & $54.4 \pm 7.7$ & $3 p$ & $34.6 \pm 5.4$ & $36.5 \pm 1.6$ \\
\hline $3 g$ & $36.4 \pm 3.8$ & $38.6 \pm 7.7$ & Myricetin $^{\mathrm{a}}$ & $28.8 \pm 6.7$ & $34.4 \pm 7.2$ \\
\hline $\mathbf{3 h}$ & $44.8 \pm 9.4$ & $45.2 \pm 1.5$ & Ningnanmycin ${ }^{\mathrm{a}}$ & $51.8 \pm 4.3$ & $58.3 \pm 2.9$ \\
\hline $3 \mathbf{i}$ & $66.8 \pm 9.8$ & $60.8 \pm 8.3$ & & & \\
\hline
\end{tabular}

Average of three replicates

a Ningnanmycin and myricetin were used for comparison of antiviral activity

Table 4 The $\mathrm{EC}_{50}$ values of $5 \mathrm{~d}, 5 \mathrm{f}, 5 \mathrm{i}$ and $5 \mathrm{~m}$ against TMV

\begin{tabular}{|c|c|c|c|c|c|}
\hline \multirow[t]{2}{*}{ Compd. } & \multicolumn{2}{|l|}{ TMV } & \multirow[t]{2}{*}{ Regression equation } & \multirow[t]{2}{*}{$r$} & \multirow[t]{2}{*}{$\mathrm{EC}_{50}(\mu \mathrm{g} / \mathrm{mL})$} \\
\hline & $500 \mu \mathrm{g} / \mathrm{mL}$ & $250 \mu \mathrm{g} / \mathrm{mL}$ & & & \\
\hline 3d & $59.8 \pm 6.2$ & $55.2 \pm 4.4$ & $y=0.473 x-3.967$ & 0.98 & $152.8 \pm 3.2$ \\
\hline $3 f$ & $68.4 \pm 7.4$ & $64.2 \pm 8.8$ & $y=0.744 x-3.512$ & 0.99 & $99.7 \pm 2.7$ \\
\hline $3 \mathbf{i}$ & $66.8 \pm 9.8$ & $63.3 \pm 5.8$ & $y=0.816 x+3.823$ & 0.99 & $127.1 \pm 2.6$ \\
\hline $3 m$ & $57.1 \pm 9.6$ & $52.3 \pm 8.5$ & $y=0.361 x+4.197$ & 0.99 & $167.3 \pm 4.8$ \\
\hline Ningnanmycin ${ }^{a}$ & $51.3+2.6$ & $50.3+3.8$ & $y=0.203 x+4.154$ & 0.97 & $211.1 \pm 3.6$ \\
\hline
\end{tabular}

Average of three replicates

a The commercial agricultural antiviral agent ningnanmycin was used for comparison of antiviral activity

2,4-di-ClPh and 2-Cl-thiazol-5-yl groups, the protective activities of corresponding compounds $3 \mathbf{c}, 3 \mathbf{i}$ and $3 \mathbf{m}$ at $500 \mu \mathrm{g} / \mathrm{mL}$ were $58.4,60.8$ and $56.7 \%$, respectively, which were similar to that of ningnanmycin (58.3\%).

\section{Methods and materials Chemistry}

The melting points of the products were determined on an XT-4 binocular microscope (Beijing Tech Instrument Co.). The ${ }^{1} \mathrm{H}$ NMR and ${ }^{13} \mathrm{C}$ NMR $\left(\mathrm{CDCl}_{3}\right.$ or DMSO as solvents) spectroscopies were performed on a JEOL-ECX 500 NMR spectrometer at room temperature using TMS as an internal standard. The IR spectra were recorded on a Bruker VECTOR 22 spectrometer using $\mathrm{KBr}$ disks. High-performance liquid chromatography mass spectrometry was performed on a Thermo Scientific Q Exactive (USA). Unless noted, all solvents and reagents were purchased from Shanghai Titan Scientific Co., Ltd, and were treated with standard methods. Based on the synthesis procedures described in our previous work [14], intermediates 1 (2-((5,7-dimethoxy4-oxo-2-(3,4,5-trimethoxyphenyl)-4H-chromen-3-yl) oxy)aceto-hydrazide) were prepared using myricetrin (5,7-dihydroxy-3-(3,4,5-trihydroxy-6-methyltetrahydro2H-pyran-2-yl)oxy)-2-(3,4,5-trihydroxyphenyl)-4Hchromen-4-one) as the starting material.

General synthesis procedure for 5,7-dimethoxy-2-(3,4,5-trimethoxyphenyl)-3- ((5-mercapto-1,3,4-thiadiazol-2-yl) methoxy)-4H-chromen-4-one (2)

To a solution of intermediate $1(1.00 \mathrm{~g}, 2.17 \mathrm{mmol})$ in methanol $(30 \mathrm{~mL})$, potassium hydroxide $(0.20 \mathrm{~mL}$, $3.16 \mathrm{mmol})$ and carbon disulfide $(0.21 \mathrm{~mL}, 3.47 \mathrm{mmol})$ were added, and the reaction mixture was heated under reflux for $16 \mathrm{~h}$. After the reaction was cooled to room temperature, $50 \mathrm{~mL}$ of water was added to the mixture, and the $\mathrm{pH}$ of the solution was adjusted to five with dilute $\mathrm{HCl}$. Then, a solid precipitated was filtered and recrystallized with ethanol to obtain the intermediate 2 . white solid, m. p. $154-155^{\circ} \mathrm{C}$, yield $50.1 \%$; IR $\left(\mathrm{KBr}, \mathrm{cm}^{-1}\right)$ : 3229 , 2939, 2837, 1639, 1634, 1608, 1575, 1498, 1466, 1357, 1253, 1211, 1130, 944, 816; ${ }^{1} \mathrm{H}$ NMR (500 MHz, DMSO$\left.d_{6}\right) \delta 7.24(\mathrm{~s}, 2 \mathrm{H}, \mathrm{Ar}-\mathrm{H}), 6.87(\mathrm{~d}, J=2.1 \mathrm{~Hz}, 1 \mathrm{H}, \mathrm{Ar}-\mathrm{H})$, $6.53(\mathrm{~d}, J=2.1 \mathrm{~Hz}, 1 \mathrm{H}, \mathrm{Ar}-\mathrm{H}), 5.09\left(\mathrm{~s}, 2 \mathrm{H}, \mathrm{CH}_{2}\right), 3.91(\mathrm{~s}$, $\left.3 \mathrm{H}, \mathrm{OCH}_{3}\right), 3.86\left(\mathrm{~s}, 9 \mathrm{H}, 3 \mathrm{OCH}_{3}\right), 3.77\left(\mathrm{~s}, 3 \mathrm{H}, \mathrm{OCH}_{3}\right) ;{ }^{13} \mathrm{C}$ NMR $\left(125 \mathrm{MHz}, \mathrm{DMSO}-d_{6}\right) \delta 183.1,176.9,169.4,165.6$, 
$164.6,163.5,158.2,157.9,145.03,143.4,129.9,113.5$, $111.2,101.5,98.6,67.3,65.5,61.5,61.4,61.3$; HRMS (HPLC) $m / z: 519.0890$, found $519.0883\left([\mathrm{M}+\mathrm{H}]^{+}\right)$.

\section{General synthesis procedures for title compounds $\mathbf{3 a - 3 p}$}

To a solution of $2(1.16 \mathrm{mmol})$ in acetonitrile $(30 \mathrm{~mL})$, sodium carbonate $(1.74 \mathrm{mmol})$ and $\mathrm{CH}_{3} \mathrm{I}(1.74 \mathrm{mmol})$ were added, and the reaction mixture was stirred at $40{ }^{\circ} \mathrm{C}$ for $5 \mathrm{~h}$. After the reaction was completed and cooled to room temperature, a solid precipitated was filtered and recrystallized with methanol to obtain the title compound 3a. Based on the similar method, the title compounds $\mathbf{3 b}-\mathbf{3} \mathbf{p}$ were prepared.

5,7-Dimethoxy-2-(3,4,5-trimethoxyphenyl)-3-((5-(methylth io)-1,3,4-thiadiazol-2-yl)methoxy)-4H-chromen-4-one (3a) A white solid, m. p. $183-184{ }^{\circ} \mathrm{C}$, yield $50.3 \%$; IR (KBr, $\left.\mathrm{cm}^{-1}\right)$ : 3006, 2957, 2839, 1645, 1616, 1580, 1474, 1427, 1417, 1212, 1163, 1158, 993, 819, 768; ${ }^{1} \mathrm{H}$ NMR $\left(500 \mathrm{MHz}, \mathrm{CDCl}_{3}\right) \delta 7.10(\mathrm{~s}, 2 \mathrm{H}, \mathrm{Ar}-\mathrm{H}), 6.47(\mathrm{~s}, 1 \mathrm{H}$, $\mathrm{Ar}-\mathrm{H}), 6.34(\mathrm{~s}, 1 \mathrm{H}, \mathrm{Ar}-\mathrm{H}), 5.23\left(\mathrm{~s}, 2 \mathrm{H}, \mathrm{CH}_{2}\right), 3.95(\mathrm{~s}, 3 \mathrm{H}$, $\left.\mathrm{OCH}_{3}\right), 3.90-3.87\left(\mathrm{~m}, 12 \mathrm{H}, 4 \mathrm{OCH}_{3}\right), 2.56\left(\mathrm{~s}, 3 \mathrm{H}, \mathrm{CH}_{3}\right)$; ${ }^{13} \mathrm{C} \mathrm{NMR}\left(125 \mathrm{MHz}, \mathrm{CDCl}_{3}\right) \delta 173.3,166.6,164.4,163.3$, $161.1,159.0,154.3,152.9,140.1,138.6,125.1,109.3$, 106.1, 96.2, 92.7, 62.3, 61.03, 56.5, 56.4, 56.9, 14.4; HRMS (HPLC) $m / z: 555.0866$, found $555.0837\left([\mathrm{M}+\mathrm{Na}]^{+}\right)$.

\section{5,7-Dimethoxy-2-(3,4,5-trimethoxyphenyl)-3- ((5-((4-nitrobenzyl)thio)-1,3,4-thiadiazol-2-yl) methoxy)-4H-chromen-4-one (3b)}

A yellow solid, m. p. $124-125{ }^{\circ} \mathrm{C}$, yield $30.1 \%$; IR (KBr, $\mathrm{cm}^{-1}$ ): 2942, 1700, 1637,1604, 1575, 1519, 1471, 1455, 1349, 1362, 1243, 1211, 1164, 1126, 1108, 1017, 856, 821; ${ }^{1} \mathrm{H}$ NMR $\left(500 \mathrm{MHz}, \mathrm{DMSO}-d_{6}\right) \delta 8.13(\mathrm{~d}, J=8.7 \mathrm{~Hz}$, $2 \mathrm{H}, \mathrm{Ar}-\mathrm{H}), 7.62(\mathrm{~d}, J=8.7 \mathrm{~Hz}, 2 \mathrm{H}, \mathrm{Ar}-\mathrm{H}), 7.18(\mathrm{~s}, 2 \mathrm{H}$, $\mathrm{Ar}-\mathrm{H}), 6.82(\mathrm{~d}, J=2.1 \mathrm{~Hz}, 1 \mathrm{H}, \mathrm{Ar}-\mathrm{H}), 6.50(\mathrm{~d}, J=2.1 \mathrm{~Hz}$, $1 \mathrm{H}, \mathrm{Ar}-\mathrm{H}), 5.21\left(\mathrm{~s}, 2 \mathrm{H}, \mathrm{CH}_{2}\right), 4.48\left(\mathrm{~s}, 2 \mathrm{H}, \mathrm{CH}_{2}\right), 3.87(\mathrm{~s}$, $\left.3 \mathrm{H}, \mathrm{OCH}_{3}\right), 3.83\left(\mathrm{~s}, 3 \mathrm{H}, \mathrm{OCH}_{3}\right), 3.77\left(\mathrm{~s}, 6 \mathrm{H}, 2 \mathrm{OCH}_{3}\right)$, $3.70\left(\mathrm{~s}, 3 \mathrm{H}, \mathrm{OCH}_{3}\right) ;{ }^{13} \mathrm{C} \mathrm{NMR}\left(125 \mathrm{MHz}, \mathrm{DMSO}-d_{6}\right)$ $\delta 172.1,164.6,164.5,164.2,160.9,158.8,153.2,153.1$, $147.4,145.1,140.2,138.6,130.8,128.5,125.2,124.6$, 124.1, 108.8, 106.4, 96.8, 93.8, 62.3, 60.7, 56.7, 56.6, 56.5, 35.2; HRMS (HPLC) m/z: 676.1030, found 676.0.0985 $\left([\mathrm{M}+\mathrm{Na}]^{+}\right)$.

\section{5,7-Dimethoxy-2-(3,4,5-trimethoxyphenyl)-3- ((5-((2-methylbenzyl)thio)-1,3,4-thiadiazol-2-yl) methoxy)-4H-chromen-4-one (3c)}

A white solid, m. p. $155-157{ }^{\circ} \mathrm{C}$, yield $54.3 \%$; IR (KBr, $\mathrm{cm}^{-1}$ ): 3010, 2954, 2838, 1649, 1610, 1572, 1511, 1470, $1452,1424,1356,1211,1194,1181,1166,1126,1058$, 1019, 978,949, 827, 817; ${ }^{1} \mathrm{H}$ NMR (500 $\left.\mathrm{MHz} \mathrm{CDCl}_{3}\right)$ $\delta 7.26(\mathrm{~s}, 1 \mathrm{H}, \mathrm{Ar}-\mathrm{H}), 7.25(\mathrm{~s}, 1 \mathrm{H}, \mathrm{Ar}-\mathrm{H}), 7.14(\mathrm{~s}, 2 \mathrm{H}$,
Ar-H), 7.11 (d, J=7.8 Hz, 2H, Ar-H), 6.49 (d, J=2.2 Hz, $1 \mathrm{H}, \mathrm{Ar}-\mathrm{H}), 6.37$ (d, J = 2.2 Hz, 1H, Ar-H), 5.27 (s, $2 \mathrm{H}$, $\mathrm{CH}_{2}$ ), 4.31 (s, $\left.2 \mathrm{H}, \mathrm{CH}_{2}\right), 3.97\left(\mathrm{~s}, 3 \mathrm{H}, \mathrm{OCH}_{3}\right), 3.91(\mathrm{~s}, 3 \mathrm{H}$, $\left.\mathrm{OCH}_{3}\right), 3.90\left(\mathrm{~s}, 3 \mathrm{H}, \mathrm{OCH}_{3}\right), 3.88\left(\mathrm{~s}, 6 \mathrm{H}, 2 \mathrm{OCH}_{3}\right), 2.31$ $\left(\mathrm{s}, 3 \mathrm{H}, \mathrm{CH}_{3}\right) ;{ }^{13} \mathrm{C} \mathrm{NMR}\left(125 \mathrm{MHz}, \mathrm{CDCl}_{3}\right) \delta 173.3,165.7$, $164.4,163.3,161.2,159.0,154.1,153.0,140.3,138.7$, 138.1, 132.0, 129.6, 129.2, 125.1, 109.4, 106.1, 96.2, 92.7, 62.4, 61.1, 56.6, 56.4, 56.0, 36.5, 29.8, 21.3; HRMS (HPLC) $\mathrm{m} / \mathrm{z}: 645.1335$, found $645.1330\left([\mathrm{M}+\mathrm{Na}]^{+}\right)$.

\section{5,7-Dimethoxy-2-(3,4,5-trimethoxyphenyl)-3- ((5-((4-chlorobenzyl)thio)-1,3,4-thiadiazol-2-yl) methoxy)-4H-chromen-4-one (3d)}

A white solid; m. p. $127-128{ }^{\circ} \mathrm{C}$; yield, $60.1 \%$; IR (KBr, $\mathrm{cm}^{-1}$ ): 3003, 2947, 2838, 1652, 1633, 1613, 1578, 1492, $1477,1469,1416,1356,1241,1212,1132,1058,1017$, 948, 839, 814; ${ }^{1} \mathrm{H}$ NMR $\left(500 \mathrm{MHz}, \mathrm{CDCl}_{3}\right) \delta 7.33(\mathrm{t}$, $J=5.7 \mathrm{~Hz}, 2 \mathrm{H}, \mathrm{Ar}-\mathrm{H}), 7.27$ (d, $J=1.6 \mathrm{~Hz}, 1 \mathrm{H}, \mathrm{Ar}-\mathrm{H})$, 7.14 (s, 2H, Ar-H), 6.50 (d, J=2.0 Hz, 1H, Ar-H), 6.38 $(\mathrm{d}, J=2.0 \mathrm{~Hz}, 1 \mathrm{H}, \mathrm{Ar}-\mathrm{H}), 5.27\left(\mathrm{~s}, 2 \mathrm{H}, \mathrm{CH}_{2}\right), 4.30(\mathrm{~s}$, $2 \mathrm{H}, \mathrm{CH}_{2}$ ), $3.98\left(\mathrm{~s}, 3 \mathrm{H}, \mathrm{OCH}_{3}\right), 3.91(\mathrm{~d}, J=2.7 \mathrm{~Hz}, 6 \mathrm{H}$, $\left.2 \mathrm{OCH}_{3}\right), 3.89\left(\mathrm{~s}, 6 \mathrm{H}, 2 \mathrm{OCH}_{3}\right) ;{ }^{13} \mathrm{C} \mathrm{NMR}(125 \mathrm{MHz}$, $\left.\mathrm{CDCl}_{3}\right) \delta 173.3,165.2,164.4,163.5,161.2,159.0,154.1$, 152.9, 140.2, 138.7, 134.1, 133.9, 130.6, 129.0, 125.1, 109.4, 106.1, 96.2, 92.7, 62.4, 61.1, 56.6, 56.4, 56.0, 35.9; HRMS (HPLC) $m / z: 665.0789$, found $665.0746\left([\mathrm{M}+\mathrm{Na}]^{+}\right)$.

5,7-Dimethoxy-2-(3,4,5-trimethoxyphenyl)-3-((5-(ethylthio)1,3,4-thiadiazol-2-yl)methoxy)-4H-chromen-4-one (3e)

A white solid, m. p. $187-188{ }^{\circ} \mathrm{C}$; yield $35.3 \%$; IR (KBr, $\mathrm{cm}^{-1}$ ): 2953, 2836, 1645, 1634, 1580, 1492, 1472, 1452, 1414, 1357, 1213, 1169, 1123, 1105, 992, 817; ${ }^{1} \mathrm{H}$ NMR $\left(500 \mathrm{MHz}, \mathrm{DMSO}-d_{6}\right) \delta 7.18(\mathrm{~s}, 2 \mathrm{H}, \mathrm{Ar}-\mathrm{H}), 6.81(\mathrm{~s}, 1 \mathrm{H}$, $\mathrm{Ar}-\mathrm{H}), 6.49$ (s, 1H, Ar-H), $5.22\left(\mathrm{~s}, 2 \mathrm{H}, \mathrm{CH}_{2}\right), 3.87$ (s, $\left.3 \mathrm{H}, \mathrm{OCH}_{3}\right), 3.83\left(\mathrm{~s}, 3 \mathrm{H}, \mathrm{OCH}_{3}\right), 3.80\left(\mathrm{~s}, 6 \mathrm{H}, 2 \mathrm{OCH}_{3}\right)$, $3.72\left(\mathrm{~s}, 3 \mathrm{H}, \mathrm{OCH}_{3}\right), 3.07\left(\mathrm{q}, J=6.8 \mathrm{~Hz}, 2 \mathrm{H}, \mathrm{CH}_{2}\right.$ ), 1.24 $\left(\mathrm{t}, J=4.5 \mathrm{~Hz}, 3 \mathrm{H}, \mathrm{CH}_{3}\right) ;{ }^{13} \mathrm{C}$ NMR (125 MHz, DMSO$\left.d_{6}\right) \delta 172.1,165.3,164.6,163.7,160.9,158.8,153.3,153.1$, 140.2, 138.5, 125.2, 108.8, 106.3, 96.7, 93.8, 62.2, 60.7, 56.7, 56.6, 56.5, 26.9, 15.1; HRMS (HPLC) $m / z: 569.1022$, found $569.0983\left([\mathrm{M}+\mathrm{Na}]^{+}\right)$.

\section{5,7-Dimethoxy-2-(3,4,5-trimethoxyphenyl)-3- ((5-((2-chlorobenzyl)thio)-1,3,4-thiadiazol-2-yl) methoxy)-4H-chromen-4-one (3f)}

A white solid, m. p. $112-113{ }^{\circ} \mathrm{C}$; yield $36.6 \%$; IR (KBr, $\left.\mathrm{cm}^{-1}\right)$ : 2997, 2942, 2838, 1636, 1603, 1578, 1572, 1505, $1490,1470,1454,1415,1350,1245,1211,1164,1127$, 1108, 1018, 1003, 853, 820; ${ }^{1} \mathrm{H}$ NMR (500 MHz, $\mathrm{CDCl}_{3}$ ) $\delta 7.52(\mathrm{~d}, J=7.4 \mathrm{~Hz}, 1 \mathrm{H}, \mathrm{Ar}-\mathrm{H}), 7.38-7.34(\mathrm{~m}, 1 \mathrm{H}$, Ar-H), 7.20 (m, 2H, Ar-H), 7.15 (s, 2H, Ar-H), 6.49 (d, $J=2.2 \mathrm{~Hz}, 1 \mathrm{H}, \mathrm{Ar}-\mathrm{H}), 6.37(\mathrm{~d}, J=2.1 \mathrm{~Hz}, 1 \mathrm{H}, \mathrm{Ar}-\mathrm{H})$, $5.28\left(\mathrm{~s}, 2 \mathrm{H}, \mathrm{CH}_{2}\right), 4.45\left(\mathrm{~s}, 2 \mathrm{H}, \mathrm{CH}_{2}\right), 3.97$ (s, 3H, $\left.\mathrm{OCH}_{3}\right)$, 
$3.91\left(\mathrm{~s}, 3 \mathrm{H}, \mathrm{OCH}_{3}\right), 3.90\left(\mathrm{~s}, 3 \mathrm{H}, \mathrm{OCH}_{3}\right), 3.87(\mathrm{~s}, 6 \mathrm{H}, 2$ $\left.\mathrm{OCH}_{3}\right) ;{ }^{13} \mathrm{C}$ NMR $\left(125 \mathrm{MHz}, \mathrm{CDCl}_{3}\right) \delta 173.3,165.5$, $164.4,163.6,161.2,159.0,154.0,153.0,140.2,138.7$, $134.4,133.5,131.6,129.8,129.7,127.2,125.1,109.4$, 106.0, 96.2, 92.6, 62.4, 61.1, 56.8, 56.4, 56.0, 34.5; HRMS (HPLC) $m / z: 665.0789$, found $665.0747\left(\left([\mathrm{M}+\mathrm{Na}]^{+}\right)\right.$.

\section{5,7-Dimethoxy-2-(3,4,5-trimethoxyphenyl)-3- ((5-((2-fluorobenzyl)thio)-1,3,4-thiadiazol-2-yl) methoxy)-4H-chromen-4-one (3g)}

A white solid, m. p. $124-125{ }^{\circ} \mathrm{C}$, yield $70.4 \%$; IR (KBr, $\left.\mathrm{cm}^{-1}\right)$ : 2975, 2942, 2842, 1637, 1604, 1492, 1470, $1455,1415,1350,1244,1212,1167,1167,1126,1106$, 1017, 1005, 855; ${ }^{1} \mathrm{H}$ NMR $\left(500 \mathrm{MHz}, \mathrm{CDCl}_{3}\right) \delta 7.44(\mathrm{t}$, $J=7.6 \mathrm{~Hz}, 1 \mathrm{H}, \mathrm{Ar}-\mathrm{H}), 7.25(\mathrm{~d}, J=1.3 \mathrm{~Hz}, 1 \mathrm{H}, \mathrm{Ar}-\mathrm{H})$, $7.14(\mathrm{~s}, 2 \mathrm{H}, \mathrm{Ar}-\mathrm{H}), 7.09-6.98(\mathrm{~m}, 2 \mathrm{H}, \mathrm{Ar}-\mathrm{H}), 6.48(\mathrm{~s}$, $1 \mathrm{H}, \mathrm{Ar}-\mathrm{H}), 6.36(\mathrm{~s}, 1 \mathrm{H}, \mathrm{Ar}-\mathrm{H}), 5.28\left(\mathrm{~s}, 2 \mathrm{H}, \mathrm{CH}_{2}\right), 4.37(\mathrm{~s}$, $\left.2 \mathrm{H}, \mathrm{CH}_{2}\right), 3.96\left(\mathrm{~s}, 3 \mathrm{H}, \mathrm{OCH}_{3}\right), 3.90\left(\mathrm{~s}, 6 \mathrm{H}, 2 \mathrm{OCH}_{3}\right), 3.87$ $\left(\mathrm{s}, 6 \mathrm{H}, 2 \mathrm{OCH}_{3}\right) ;{ }^{13} \mathrm{C} \mathrm{NMR}\left(125 \mathrm{MHz}, \mathrm{CDCl}_{3}\right) \delta 173.3$, $165.4,164.4,163.5,161.2,160.3,159.8,159.0,154.1$, $153.0,140.3,138.7,131.5,130.2,125.1,124.4,122.8$, $115.8,115.6,109.4,106.1,96.2,92.7,62.4,61.0,56.5$, 56.0, 29.9; HRMS (HPLC) $m / z: 649.1085$, found 649.1046 $\left([\mathrm{M}+\mathrm{Na}]^{+}\right)$.

\section{5,7-Dimethoxy-2-(3,4,5-trimethoxyphenyl)-3- ((5-((4-methoxybenzyl)thio)-1,3,4-thiadiazol-2-yl) methoxy)-4H-chromen-4-one (3h)}

A white solid, m. p. $146-147{ }^{\circ} \mathrm{C}$, yield $35.7 \%$; IR (KBr, $\left.\mathrm{cm}^{-1}\right)$ : 2950, 1755, 1645, 1629, 1604, 1507, 1492, 1457, $1430,1410,1354,1249,1210,1180,1161,1129,1112$, 1064, 1016, 841, 816; ${ }^{1} \mathrm{H}$ NMR (500 MHz, $\mathrm{CDCl}_{3}$ ) $\delta 7.27$ $(\mathrm{d}, J=8.1 \mathrm{~Hz}, 2 \mathrm{H}, \mathrm{Ar}-\mathrm{H}), 7.19(\mathrm{~s}, 1 \mathrm{H}, \mathrm{Ar}-\mathrm{H}), 6.83(\mathrm{~d}$, $J=7.5 \mathrm{~Hz}, 4 \mathrm{H}, \mathrm{Ar}-\mathrm{H}), 6.50(\mathrm{~s}, 1 \mathrm{H}, \mathrm{Ar}-\mathrm{H}), 5.23(\mathrm{~s}, 2 \mathrm{H}$, $\left.\mathrm{CH}_{2}\right), 4.29\left(\mathrm{~s}, 2 \mathrm{H}, \mathrm{CH}_{2}\right), 3.87\left(\mathrm{~s}, 3 \mathrm{H}, \mathrm{OCH}_{3}\right), 3.83(\mathrm{~s}, 3 \mathrm{H}$, $\left.\mathrm{OCH}_{3}\right), 3.78\left(\mathrm{~s}, 6 \mathrm{H}, 2 \mathrm{OCH}_{3}\right), 3.70\left(\mathrm{~s}, 3 \mathrm{H}, \mathrm{OCH}_{3}\right), 3.69$ $\left(\mathrm{s}, 3 \mathrm{H}, \mathrm{OCH}_{3}\right) ;{ }^{13} \mathrm{C}$ NMR $\left(125 \mathrm{MHz}, \mathrm{CDCl}_{3}\right) \delta 172.2$, $167.0,164.6,163.9,160.9,159.4,158.8,153.1,140.2$, 138.6, 130.9, 128.4, 125.3, 114.5, 114.0, 108.8, 106.4, 96.7, 93.8, 63.1, 62.3, 60.7, 56.6, 55.6, 35.9; HRMS (HPLC) $m / z$ : 639.1447, found 639.1444 $\left([\mathrm{M}+\mathrm{H}]^{+}\right)$.

\section{5,7-Dimethoxy-2-(3,4,5-trimethoxyphenyl)-3-((5 -((2,4-dichlorobenzyl)thio)-1,3,4-thiadiazol-2-yl) methoxy)-4H-chromen-4-one (3i)}

A white solid, m. p. $154-155{ }^{\circ} \mathrm{C}$, yield $90.1 \%$; IR $(\mathrm{KBr}$, $\left.\mathrm{cm}^{-1}\right)$ : 2944, 1643, 1616, 1571, 1460, 1416, 1355, 1242, 1216, 1162, 1135, 1058, 1018, 955, 827; ${ }^{1} \mathrm{H}$ NMR $\left(500 \mathrm{MHz}, \mathrm{CDCl}_{3}\right) \delta 7.51(\mathrm{~d}, J=8.3 \mathrm{~Hz}, 1 \mathrm{H}, \mathrm{Ar}-\mathrm{H}), 7.38$ $(\mathrm{d}, J=2.1 \mathrm{~Hz}, 1 \mathrm{H}, \mathrm{Ar}-\mathrm{H}), 7.17(\mathrm{~d}, J=8.3 \mathrm{~Hz}, 1 \mathrm{H}, \mathrm{Ar}-\mathrm{H})$, $7.14(\mathrm{~s}, 2 \mathrm{H}, \mathrm{Ar}-\mathrm{H}), 6.50$ (d, $J=2.1 \mathrm{~Hz}, 1 \mathrm{H}, \mathrm{Ar}-\mathrm{H}), 6.38$ $(\mathrm{d}, J=2.1 \mathrm{~Hz}, 1 \mathrm{H}, \mathrm{Ar}-\mathrm{H}), 5.28\left(\mathrm{~s}, 2 \mathrm{H}, \mathrm{CH}_{2}\right), 4.40(\mathrm{~s}, 2 \mathrm{H}$, $\mathrm{CH}_{2}$ ), $3.98\left(\mathrm{~s}, 3 \mathrm{H}, \mathrm{OCH}_{3}\right), 3.91\left(\mathrm{~s}, 6 \mathrm{H}, 2 \mathrm{OCH}_{3}\right), 3.88$ (s, $\left.6 \mathrm{H}, 2 \mathrm{OCH}_{3}\right) ;{ }^{13} \mathrm{C} \mathrm{NMR}\left(125 \mathrm{MHz}, \mathrm{CDCl}_{3}\right) \delta 173.3,165.2$, $164.4,163.7,161.2,159.0,154.0,153.0,138.7,135.1$, 134.9, 132.4, 132.2, 129.6, 127.5, 125.1, 109.4, 106.1, 96.2, 92.7, 62.4, 61.1, 56.6, 56.4, 56.0, 33.8; HRMS (HPLC) m/z: 699.0399, found $699.0365\left([\mathrm{M}+\mathrm{Na}]^{+}\right)$.

\section{5,7-Dimethoxy-2-(3,4,5-trimethoxyphenyl)-3- ((5-((3-nitrobenzyl)thio)-1,3,4-thiadiazol-2-yl) methoxy)-4H-chromen-4-one (3j)}

A white solid, m. p. $180-181{ }^{\circ} \mathrm{C}$, yield $50.5 \%$; $\mathrm{IR}(\mathrm{KBr}$, $\mathrm{cm}^{-1}$ ): 2942, 1700, 1637, 1604, 1575, 1519, 1471, 1455, $1349,1362,1243,1211,1164,1126,1108,1017,856,821$; ${ }^{1} \mathrm{H}$ NMR $\left(500 \mathrm{MHz}, \mathrm{CDCl}_{3}\right) \delta 8.10(\mathrm{~d}, J=8.1 \mathrm{~Hz}, 1 \mathrm{H}$, $\mathrm{Ar}-\mathrm{H}), 7.75(\mathrm{~d}, J=7.6 \mathrm{~Hz}, 1 \mathrm{H}, \mathrm{Ar}-\mathrm{H}), 7.56(\mathrm{t}, J=7.5 \mathrm{~Hz}$, $1 \mathrm{H}, \mathrm{Ar}-\mathrm{H}), 7.49-7.43(\mathrm{~m}, 1 \mathrm{H}, \mathrm{Ar}-\mathrm{H}), 7.14(\mathrm{~s}, 2 \mathrm{H}, \mathrm{Ar}-\mathrm{H})$, $6.50(\mathrm{~d}, J=2.1 \mathrm{~Hz}, 1 \mathrm{H}, \mathrm{Ar}-\mathrm{H}), 6.37(\mathrm{~d}, J=2.2 \mathrm{~Hz}$, $1 \mathrm{H}, \mathrm{Ar}-\mathrm{H}), 5.27\left(\mathrm{~s}, 2 \mathrm{H}, \mathrm{CH}_{2}\right), 4.68\left(\mathrm{~s}, 2 \mathrm{H}, \mathrm{CH}_{2}\right), 3.97(\mathrm{~s}$, $\left.3 \mathrm{H}, \mathrm{OCH}_{3}\right), 3.91\left(\mathrm{~s}, 3 \mathrm{H}, \mathrm{OCH}_{3}\right), 3.89\left(\mathrm{~s}, 3 \mathrm{H}, \mathrm{OCH}_{3}\right), 3.86$ $\left(\mathrm{s}, 6 \mathrm{H}, 2 \mathrm{OCH}_{3}\right) ;{ }^{13} \mathrm{C}$ NMR $\left(125 \mathrm{MHz}, \mathrm{CDCl}_{3}\right) \delta 173.3$, $165.7,164.4,163.8,161.2,159.0,154.0,153.0,147.6$, $140.3,138.8,134.1,133.1,132.5,129.4,125.7,125.1,109.4$, 106.1, 96.2, 92.7, 62.4, 61.0, 56.6, 56.4, 56.0, 34.2; HRMS (HPLC) $m / z: 676.1030$, found $676.1012\left([\mathrm{M}+\mathrm{Na}]^{+}\right)$.

\section{5,7-Dimethoxy-2-(3,4,5-trimethoxyphenyl)-3- ((5-((4-bromobenzyl)thio)-1,3,4-thiadiazol-2-yl) methoxy)-4H-chromen-4-one (3k)}

A white solid, m. p. $131-132{ }^{\circ} \mathrm{C}$; yield, $39.4 \%$; IR $(\mathrm{KBr}$, $\left.\mathrm{cm}^{-1}\right)$ : 2945, 1634, 1605, 1558, 1471, 1426, 1352, 1246, 1212, 1163, 1130, 1018, 820; ${ }^{1} \mathrm{H}$ NMR (500 MHz, $\left.\mathrm{CDCl}_{3}\right)$ $\delta 7.43(\mathrm{~d}, J=8.3 \mathrm{~Hz}, 2 \mathrm{H}, \mathrm{Ar}-\mathrm{H}), 7.28(\mathrm{~s}, 1 \mathrm{H}, \mathrm{Ar}-\mathrm{H}), 7.25$ $(\mathrm{s}, 1 \mathrm{H}, \mathrm{Ar}-\mathrm{H}), 7.13(\mathrm{~s}, 2 \mathrm{H}, \mathrm{Ar}-\mathrm{H}), 6.49(\mathrm{~d}, J=2.2 \mathrm{~Hz}$, $1 \mathrm{H}, \mathrm{Ar}-\mathrm{H}), 6.38(\mathrm{~d}, J=2.2 \mathrm{~Hz}, 1 \mathrm{H}, \mathrm{Ar}-\mathrm{H}), 5.26(\mathrm{~s}, 2 \mathrm{H}$, $\left.\mathrm{CH}_{2}\right), 4.27\left(\mathrm{~s}, 2 \mathrm{H}, \mathrm{CH}_{2}\right), 3.98\left(\mathrm{~s}, 3 \mathrm{H}, \mathrm{OCH}_{3}\right), 3.91(\mathrm{~s}, 6 \mathrm{H}$, $\left.2 \mathrm{OCH}_{3}\right), 3.88\left(\mathrm{~s}, 6 \mathrm{H}, 2 \mathrm{OCH}_{3}\right) ;{ }^{13} \mathrm{C}$ NMR $(125 \mathrm{MHz}$, $\left.\mathrm{CDCl}_{3}\right) \delta 173.3,165.2,164.4,163.5,161.2,159.0,154.1$, $152.9,140.2,138.7,134.4,132.0,131.0,125.1,122.3,109.4$, 106.1, 96.2, 92.7, 62.4, 61.1, 56.6, 56.4, 56.0, 35.9; HRMS (HPLC) $m / z: 709.0293$, found $709.0237\left([\mathrm{M}+\mathrm{Na}]^{+}\right)$.

\section{5,7-Dimethoxy-2-(3,4,5-trimethoxyphenyl)-3- ((5-((2-bromobenzyl)thio)-1,3,4-thiadiazol-2-yl) methoxy)-4H-chromen-4-one (3l)}

A white solid, m. p. $116-117{ }^{\circ} \mathrm{C}$, yield $45.4 \%$; IR $(\mathrm{KBr}$, $\mathrm{cm}^{-1}$ ): 3004, 2943, 1633, 1603, 1560, 1545, 1492, 1467, $1428,1416,1353,1247,1213,1166,1112,1126,1109$, 1018, 1005, 862, 815; ${ }^{1} \mathrm{H}$ NMR (500 MHz, $\left.\mathrm{CDCl}_{3}\right) \delta$ 7.57-7.52 (m, 2H, Ar-H), $7.23(\mathrm{t}, J=7.5 \mathrm{~Hz}, 1 \mathrm{H}, \mathrm{Ar}-\mathrm{H})$, 7.16-7.11 (m, 3H, Ar-H), 6.49 (d, $J=2.2 \mathrm{~Hz}, 1 \mathrm{H}, \mathrm{Ar}-\mathrm{H})$, $6.37(\mathrm{~d}, J=2.2 \mathrm{~Hz}, 1 \mathrm{H}, \mathrm{Ar}-\mathrm{H}), 5.28\left(\mathrm{~s}, 2 \mathrm{H}, \mathrm{CH}_{2}\right), 4.46(\mathrm{~s}$, $\left.2 \mathrm{H}, \mathrm{CH}_{2}\right), 3.97\left(\mathrm{~s}, 3 \mathrm{H}, \mathrm{OCH}_{3}\right), 3.90(\mathrm{~d}, J=1.0 \mathrm{~Hz}, 6 \mathrm{H}$, $\left.2 \mathrm{OCH}_{3}\right), 3.87\left(\mathrm{~s}, 6 \mathrm{H}, 2 \mathrm{OCH}_{3}\right) ;{ }^{13} \mathrm{C} \mathrm{NMR}(125 \mathrm{MHz}$, $\left.\mathrm{CDCl}_{3}\right) \delta 172.2,164.6,164.4,164.2,160.9,158.8,153.3$, 
$153.1,140.1,138.6,135.5,133.4,132.0,130.7,128.6$, 125.3, 124.5, 108.8, 106.4, 96.7, 93.8, 62.3, 60.7, 56.7, 56.6, 56.5, 37.1; HRMS (HPLC) $m / z: 709.0284$, found 709.0246 $\left([\mathrm{M}+\mathrm{Na}]^{+}\right)$.

\section{5,7-Dimethoxy-2-(3,4,5-trimethoxyphenyl)-3-((5-(((2-chlo- rothiazol-5-yl)methyl)thio)-1,3,4-thiadiazol-2-yl) methoxy)-4H-chromen-4-one (3m)}

A white solid, m. p. $120-121{ }^{\circ} \mathrm{C}$, yield $58.3 \%$; IR (KBr, $\mathrm{cm}^{-1}$ ): 2996, 2945, 1645, 1634, 1606, 1572, 1506, 1484, $1456,1414,1352,1242,1212,1164,1130,1106,1050$, 870, 821; ${ }^{1} \mathrm{H}$ NMR (500 MHz, DMSO- $\left.d_{6}\right) \delta 7.56(\mathrm{~s}, 1 \mathrm{H}$, Ar-H), 7.19 (s, 2H, Ar-H), 6.83 (s, 1H, Ar-H), 6.50 (s, $1 \mathrm{H}, \mathrm{Ar}-\mathrm{H}), 5.24\left(\mathrm{~s}, 2 \mathrm{H}, \mathrm{CH}_{2}\right), 4.61\left(\mathrm{~s}, 2 \mathrm{H}, \mathrm{CH}_{2}\right), 3.87(\mathrm{~s}$, $\left.3 \mathrm{H}, \mathrm{OCH}_{3}\right), 3.83\left(\mathrm{~s}, 3 \mathrm{H}, \mathrm{OCH}_{3}\right), 3.78\left(\mathrm{~s}, 6 \mathrm{H}, 2 \mathrm{OCH}_{3}\right)$, $3.69\left(\mathrm{~s}, 3 \mathrm{H}, \mathrm{OCH}_{3}\right) ;{ }^{13} \mathrm{C} \mathrm{NMR}\left(125 \mathrm{MHz}, \mathrm{DMSO}-d_{6}\right)$ $\delta 172.1,164.6,164.5,164.4,160.9,158.8,153.3,153.1$, $151.1,141.8,140.1,138.6,137.8,125.2,108.8,106.4,96.8$, 93.8, 62.3, 60.7, 56.7, 56.6, 56.5, 28.4; HRMS (HPLC) $m / z$ : 672.0306, found $672.0262\left([\mathrm{M}+\mathrm{Na}]^{+}\right)$.

5,7-Dimethoxy-2-(3,4,5-trimethoxyphenyl)-3-((5-(benzylthi o)-1,3,4-thiadiazol-2-yl)methoxy)-4H- chromen-4-one (3n) A white solid, m. p. $160-161{ }^{\circ} \mathrm{C}$, yield $35.7 \%$; IR (KBr, $\mathrm{cm}^{-1}$ ): 2979, 2942, 1634, 1602, 1579, 1505, 1492, 1470, 1454, 1416, 1351, 1246, 1211, 1163, 1128, 1108, 1000, 823; ${ }^{1} \mathrm{H}$ NMR $\left(500 \mathrm{MHz}, \mathrm{DMSO}-d_{6}\right) \delta 7.34(\mathrm{~d}, J=6.9 \mathrm{~Hz}$, $2 \mathrm{H}, \mathrm{Ar}-\mathrm{H}), 7.25$ (d, $J=10.3 \mathrm{~Hz}, 3 \mathrm{H}, \mathrm{Ar}-\mathrm{H}), 7.18(\mathrm{~s}, 2 \mathrm{H}$, $\mathrm{Ar}-\mathrm{H}), 6.82(\mathrm{t}, J=4.6 \mathrm{~Hz}, 1 \mathrm{H}, \mathrm{Ar}-\mathrm{H}), 6.49(\mathrm{~d}, J=2.1 \mathrm{~Hz}$, $1 \mathrm{H}, \mathrm{Ar}-\mathrm{H}), 5.22\left(\mathrm{~s}, 2 \mathrm{H}, \mathrm{CH}_{2}\right), 4.34\left(\mathrm{~s}, 2 \mathrm{H}, \mathrm{CH}_{2}\right), 3.87(\mathrm{~s}$, $\left.3 \mathrm{H}, \mathrm{OCH}_{3}\right), 3.83\left(\mathrm{~s}, 3 \mathrm{H}, \mathrm{OCH}_{3}\right), 3.79(\mathrm{~d}, J=13.8 \mathrm{~Hz}$, $\left.6 \mathrm{H}, 2 \mathrm{OCH}_{3}\right), 3.70\left(\mathrm{~d}, J=7.8 \mathrm{~Hz}, 3 \mathrm{H}, \mathrm{OCH}_{3}\right) ;{ }^{13} \mathrm{C} \mathrm{NMR}$ $\left(125 \mathrm{MHz}, \mathrm{CDCl}_{3}\right) \delta 172.2,164.9,164.6,164.0,160.9$, $158.8,153.3,153.1,140.1,138.6,136.6,129.5,129.1$, $128.4,125.3,108.8,106.4,96.7,93.8,62.3,60.7,56.7,56.6$, $56.5,36.1$; HRMS (HPLC) $m / z: 631.1179$, found 631.1143 $\left([\mathrm{M}+\mathrm{Na}]^{+}\right)$.

\section{5,7-Dimethoxy-2-(3,4,5-trimethoxyphenyl)-3- ((5-((4-methylbenzyl)thio)-1,3,4-thiadiazol-2-yl) methoxy)-4H-chromen-4-one (30)}

A white solid, m. p. $166-167{ }^{\circ} \mathrm{C}$, yield $28.7 \%$; IR (KBr, $\left.\mathrm{cm}^{-1}\right)$ : 2933, 2838, 1649, 1610, 1578, 1511, 1470, 1410, 1357, 1239, 1121, 1160, 1126, 1019, 938, 817; ${ }^{1} \mathrm{H}$ NMR $\left(500 \mathrm{MHz}, \mathrm{DMSO}-d_{6}\right) \delta 7.23(\mathrm{~s}, 1 \mathrm{H}, \mathrm{Ar}-\mathrm{H}), 7.21(\mathrm{~s}, 1 \mathrm{H}$, Ar-H), 7.18 (s, 2H, Ar-H), 7.07 (d, J = 7.9 Hz, 2H, Ar-H), $6.80(\mathrm{~d}, \mathrm{~J}=2.2 \mathrm{~Hz}, 1 \mathrm{H}, \mathrm{Ar}-\mathrm{H}), 6.48(\mathrm{~d}, \mathrm{~J}=2.2 \mathrm{~Hz}, 1 \mathrm{H}$, $\mathrm{Ar}-\mathrm{H}), 5.23\left(\mathrm{~s}, 2 \mathrm{H}, \mathrm{CH}_{2}\right), 4.29\left(\mathrm{~s}, 2 \mathrm{H}, \mathrm{CH}_{2}\right), 3.86(\mathrm{~s}, 3 \mathrm{H}$, $\left.\mathrm{OCH}_{3}\right), 3.82\left(\mathrm{~s}, 3 \mathrm{H}, \mathrm{OCH}_{3}\right), 3.78\left(\mathrm{~s}, 6 \mathrm{H}, 2 \mathrm{OCH}_{3}\right), 3.70$ $\left(\mathrm{s}, 3 \mathrm{H}, \mathrm{OCH}_{3}\right), 2.22\left(\mathrm{~s}, 3 \mathrm{H}, \mathrm{CH}_{3}\right) ;{ }^{13} \mathrm{C}$ NMR $(125 \mathrm{MHz}$, DMSO- $\left.d_{6}\right) \delta 172.1,164.9,164.5,163.9,160.9,158.7$, $153.3,153.1,140.2,138.6,137.7,133.4,129.6,129.4$, 125.3, 108.8, 106.4, 96.7, 93.8, 62.3, 60.7, 56.7, 56.6, 56.5,
36.0, 21.2; HRMS (HPLC) $m / z: 645.1335$, found 645.1300 $\left([\mathrm{M}+\mathrm{Na}]^{+}\right)$.

5,7-Dimethoxy-2-(3,4,5-trimethoxyphenyl)-3-((5 -((pyridin-3-ylmethyl)thio)-1,3,4-thiadiazol-2-yl) methoxy)-4H-chromen-4-one (3p)

A white solid, m. p. $155-156{ }^{\circ} \mathrm{C}$, yield $60.1 \%$; IR $(\mathrm{KBr}$, $\left.\mathrm{cm}^{-1}\right)$ : 2943, 2839, 1633, 1622, 1602, 1505, 1470, 1464, $1428,1351,1247,1212,1166,1128,1109,856,817 ;{ }^{1} \mathrm{H}$ NMR (500 MHz, DMSO- $\left.d_{6}\right) \delta 8.56(\mathrm{~s}, 1 \mathrm{H}, \mathrm{Ar}-\mathrm{H}), 8.43$ $(\mathrm{d}, J=4.5 \mathrm{~Hz}, 1 \mathrm{H}, \mathrm{Ar}-\mathrm{H}), 7.77(\mathrm{~d}, J=7.5 \mathrm{~Hz}, 1 \mathrm{H}, \mathrm{Ar}-\mathrm{H})$, 7.35-7.24 (m, 1H, Ar-H), 7.18 (s, 2H, Ar-H), 6.82 (s, 1H, $\mathrm{Ar}-\mathrm{H}), 6.50$ (s, 1H, Ar-H), $5.21\left(\mathrm{~s}, 2 \mathrm{H}, \mathrm{CH}_{2}\right), 4.38(\mathrm{~s}, 2 \mathrm{H}$, $\left.\mathrm{CH}_{2}\right), 3.87\left(\mathrm{~s}, 3 \mathrm{H}, \mathrm{OCH}_{3}\right), 3.83\left(\mathrm{~s}, 3 \mathrm{H}, \mathrm{OCH}_{3}\right), 3.77$ (s, $6 \mathrm{H}, 2$ $\left.\mathrm{OCH}_{3}\right), 3.70\left(\mathrm{~s}, 3 \mathrm{H}, \mathrm{OCH}_{3}\right) ;{ }^{13} \mathrm{C}$ NMR (125 MHz, DMSO$\left.d_{6}\right) \delta 172.1,164.6,164.6,164.1,160.9,158.8,153.3,153.1$, $150.5,149.4,140.1,138.6,137.1,133.0,125.3,124.1,108.8$, 106.4, 96.7, 93.8, 62.3, 60.7, 56.7, 56.6, 56.5, 33.3; HRMS (HPLC) $m / z: 632.1131$, found $632.1095\left([\mathrm{M}+\mathrm{Na}]^{+}\right)$.

\section{Conclusions}

Aiming to discover novel myricetin analogues with potent activities, a series of novel myricetin derivatives containing 1,3,4-thiadiazole moiety were synthesized, and their antibacterial activities against Xoo and $R s$ and their antiviral activity against TMV were evaluated. Bioassays indicated that some target compounds exhibited potential antibacterial and antiviral activities. Among them, compounds $\mathbf{2}$, 3a, 3b, 3d, 3f, 3i, 3m and $\mathbf{3 p}$ exhibited excellent antibacterial activities against Xoo, with $\mathrm{EC}_{50}$ values of $42.7,38.6,20.8,12.9,22.7,27.3,18.3$ and $29.4 \mu \mathrm{g} / \mathrm{mL}$, respectively, which were better than that of thiadiazole-copper $(94.9 \mu \mathrm{g} / \mathrm{mL})$. Compounds $\mathbf{3 b}$, 3d, 3e, 3f, 3i and 3o showed good antibacterial activities against $R s$, with $\mathrm{EC}_{50}$ values of 37.9 , 72.6, 43.6, 59.6, 60.6 and $39.6 \mu \mathrm{g} / \mathrm{mL}$, respectively, which were superior to that of thiadiazole-copper $(131.7 \mu \mathrm{g} / \mathrm{mL})$. In addition, compounds 3d, 3f, $\mathbf{3 i}$ and $\mathbf{3 m}$ showed better curative activities against $\mathrm{TMV}$, with $\mathrm{EC}_{50}$ values of 152.8, 99.7, 127.1 , and $167.3 \mu \mathrm{g} / \mathrm{mL}$, respectively, which were better than that of ningnanmycin $(211.1 \mu \mathrm{g} / \mathrm{mL})$. Given the above results, this kind of myricetin analogues could be further studied as potential alternative templates in the search for novel antibacterial and antiviral agents.

\section{Additional file}

Additional file 1. All the copies of $I R,{ }^{1} H$ NMR, ${ }^{13} \mathrm{C}$ NMR and HRMS for the title compounds.

\section{Authors' contributions}

The current study is an outcome of constructive discussion with WX. XZ, XW, $L C$ and $X R$ carry out their synthesis and characterization experiments; $X Z$, $X W, Q L, J Z$ and $C Z$ performed the antiviral and antibacterial activities; XW, XZ, 
$\mathrm{LC}$ and $\mathrm{QL}$ carried out the ${ }^{1} \mathrm{H} N M R,{ }^{13} \mathrm{C} N M R$, IR and HRMS spectral analyses; $W X$ and $X W$ were involved in the drafting of the manuscript and revising the manuscript. All authors read and approved the final manuscript.

\section{Author details}

${ }^{1}$ State Key Laboratory Breeding Base of Green Pesticide and Agricultural Bioengineering, Key Laboratory of Green Pesticide and Agricultural Bioengineering, Ministry of Education, Guizhou University, Guiyang 550025, China. ${ }^{2}$ Key Laboratory of Monitoring and Management of Crop Diseases and Pest Insects, Ministry of Agriculture, Nanjing Agricultural University, Nanjing 210095, China.

\section{Competing interests}

The authors declare that they have no competing interests.

\section{Availability of data and materials}

We have presented all our main data in the form of tables and figures. Meanwhile, all the copies of IR, ${ }^{1} \mathrm{H}$ NMR, ${ }^{13} \mathrm{C}$ NMR and HRMS for the title compounds were presented in the Additional file 1. The datasets supporting the conclusions of the article are included within the article and the Additional file 1.

\section{Consent for publication}

This section are not applicable for this manuscript.

\section{Ethics approval and consent to participate}

This section are not applicable for this manuscript.

\section{Funding and acknowledgements}

The authors gratefully acknowledge Grants from the National Key Research and Development Program of China (No. 2017YFD0200506), the National Nature Science Foundation of China (No. 21462012) and the special fund for outstanding Scientific and Technological Candidates of Guizhou Province (Nos. 2015035, 2013041)

\section{Publisher's Note}

Springer Nature remains neutral with regard to jurisdictional claims in published maps and institutional affiliations.

Received: 12 July 2017 Accepted: 11 October 2017

Published online: 17 October 2017

\section{References}

1. Xu WM, Han FF, He M, Hu DY, He J, Yang S, Song BA (2012) Inhibition of tobacco bacterial wilt with sulfone derivatives containing an 1,3,4-oxadiazole moiety. J Agric Food Chem 60:1036-1041

2. Wang PY, Zhou L, Zhou J, Wu ZB, Xue W, Song BA, Yang S (2016) Synthesis and antibacterial activity of pyridinium-tailored 2,5-substituted-1,3,4oxadiazole thioether/sulfoxide/sulfone derivatives. Bioorgan Med Chem Lett 26:1214-1217

3. Wang X, Li P, Li Z, Yin J, He M, Xue W, Chen Z, Song B (2013) Synthesis and bioactivity evaluation of novel arylimines containing a 3-aminoethyl-2[( $\rho$-trifluoromethoxy)anilino] -4(3H)-quinazolinone moiety. J Agric Food Chem 61:9575-9582

4. Chen MH, Wang XB, Tang BC, Zhang X (2016) Synthesis and antibacterial evaluation of novel Schiff base derivatives containing $4(3 \mathrm{H})$-quinazolinone moiety. Chem Pap 70:1521-1528

5. Qian X, Lee PW, Cao S (2010) China: forward to the green pesticides via a basic research program. J Agric Food Chem 58:2613-2623

6. Wang PY, Chen L, Zhou J, Fang HS, Wu ZB, Song BA, Yang S (2017) Synthesis and bioactivities of 1-ary-4-hydroxy-1H-pyrrol-2(5H)-one derivatives bearing 1,3,4-oxadiazole moiety. J Saudi Chem Soc 21:315-323

7. Naz S, Siddiqi R, Ahmad S, Rasool S, Sayeed SJ (2007) Antibacterial activity directed isolation of compounds from punica granatum. J Food Sci 72:341-345

8. Yu MS, Lee J, Lee JM, Kim Y, Chin YW, Jee JG, Keum YS, Jeong YJ (2012) Identification of myricetin and scutellarein as novel chemical inhibitors of the SARS coronavirus helicase, nsP13. Bioorgan Med Chem Lett 22:4049-4054
9. Phillips P, Sangwan V, Cacho DB, Dudeja V, Vickers S, Saluja A (2011) Myricetin induces pancreatic cancer cell death via the induction of apoptosis and inhibition of the phosphatidylinositol 3-kinase (PI3K) signaling pathway. Cancer Lett 308:181-188

10. Kim HH, Kim DH, Kim MH, Oh MH, Kim SR, Park KJ, Lee MW (2013) Flavonoid constituents in the leaves of Myrica rubra sieb. et zucc. with anti-inflammatory. Arch Pharmacal Res 36:1533-1540

11. Chobot V, Hadacek F (2011) Exploration of pro-oxidant and antioxidant activities of the flavonoid myricetin. Redox Rep 16:242-247

12. Liu IM, Liou SS, Lan TW, Hsu FL, Cheng JT (2005) Myricetin as the active principle of Abelmoschus moschatus to lower plasma glucose in streptozotocin induced disbetic. Planta Med 71:617-621

13. Zhao HJ, Zhang X, Wang ZB, Chen Y, Xia LJ, Gong HY, Xue W (2014) The synergism of extracts from bark of Toona sinensis and ningnanmycin. Agrochemicals 53:142-144

14. Xue W, Song BA, Zhao HJ, Qi XB, Huang YJ, Liu XH (2015) Novel myricetin derivatives: design, synthesis and anticancer activity. Eur J Med Chem 97:155-163

15. Xiao W, Ruan XH, Li Q, Zhang JP, Zhong XM, Xie Y, Wang XB, Huang MG, Xue W (2017) Synthesis and antibacterial activities of myricetin derivatives containing acidamide moiety. Chem J Chin Univ 38:35-40

16. Li P, Shi L, Gao MN, Yang X, Xue W, Jin LH, Hu DY, Song BA (2015) Antibacterial activities against rice bacterial leaf blight and tomato bacterial wilt of 2-mercapto-5-substituted-1,3,4-oxadiazole/thiadiazole derivatives Bioorgan Med Chem Lett 25:481-484

17. Liu F, Luo XQ, Song BA, Bhadury PS, Yang S, Jin LH, Xue W, Hu DY (2008) Synthesis and antifungal activity of novel sulfoxide derivatives containing trimethoxyphenyl substituted 1,3,4-thiadiazole and 1,3,4-oxadiazole moiety. Bioorgan Med Chem 16:3632-3640

18. Xu WM, Li SZ, He M, Yang S, Li XY, Li P (2013) Synthesis and bioactivities of novel thioether/sulfone derivatives containing 1,2,3-thiadiazole and 1,3,4-oxadiazole/thiadiazole moiety. Bioorgan Med Chem Lett 23:5821-5824

19. Rajak H, Deshmukh R, Aggarwal N, Kashaw S, Kharya MD, Mishra P (2009) Synthesis of novel 2,5-disubstituted 1,3,4-thiadiazoles for their potential anticonvulsant activity: pharmacophoric model studies. Arch Pharm Chem Life Sci 342:453-461

20. Clerici F, Pocar D, Guido M, Loche A, Perlini V, Brufani M (2001) Synthesis of 2-amino-5-sulfanyl-1,3,4-thiadiazole derivatives and evaluation of their antidepressant and anxiolytic activity. J Med Chem 44:931-936

21. Altintop MD, Can OD, Ozkay VD, Kaplancikli ZA (2016) Synthesis and evaluation of new 1,3,4-thiadiazole derivatives as antinociceptive agents. Molecules 21:1004-1013

22. Flefel EM, El-Sayed WA, Mohamed AM, El-Sofany WI, Awad HM (2017) Synthesis and anticancer activity of new 1-thia-4-azaspiro[4.5] decane, their derived thiazolopyrimidine and 1,3,4-thiadiazole thioglycosides. Molecules 22:170-182

23. Li P, Shi L, Yang X, Yang L, Chen XW, Wu F, Shi QC, Xu WM, He M, Hu DY, Song BA (2014) Design, synthesis, and antibacterial activity against rice bacterial leaf blight and leaf streak of 2,5-substituted-1,3,4-oxadiazole/ thiadiazole sulfone derivative. Bioorgan Med Chem Lett 24:1677-1680

24. Yu L, Gan X, Zhou D, He F, Zeng S, Hu D (2017) Synthesis and antiviral activity of novel 1,4-pentadien-3-one derivatives containing a 1,3,4-thiadiazole moiety. Molecules 22:658-666

25. Ma J, Li P, Li X, Shi Q, Wan Z, Hu D, Jin L, Song B (2014) Synthesis and antiviral bioactivity of novel 3-((2-((1E,4E)-3-oxo-5-arylpenta-1,4-dien-1-yl) phenoxy)methyl)-4(3H)-quinazolin-one derivatives. J Agric Food Chem 62:8928-8934

26. Long C, Li P, Chen M, Dong L, Hu D, Song B (2015) Synthesis, anti-tobacco mosaic virus and cucumber mosaic virus activity, and 3D-QSAR study of novel 1,4-pentadien-3-one derivatives containing 4-thioquinazoline moiety. Eur J Med Chem 102:639-647

27. Gan X, Hu D, Li P, Wu J, Chen X, Xue W, Song B (2016) Design, synthesis, antiviral activity and three-dimensional quantitative structure-activity relationship study of novel 1,4-pentadien-3-one derivatives containing the 1,3,4-oxadiazole moiety. Pest Manag Sci 72:534-543 